\title{
Do Tanzanian hospitals need healthcare ethics committees? Report on the 2014 Dartmouth/Penn Research Ethics Training and Program Development for Tanzania (DPRET) workshop
}

\author{
M Aboud, ${ }^{1}$ MD, MMed, MSC (Urol); D Bukini, ${ }^{2}$ MBE, MPH; R Waddell, ${ }^{3}$ DSC; L Peterson, ${ }^{4}$ MD; R Joseph, ${ }^{5}$ MD, MMed (Paediatr), MBE; \\ B M Morris, ${ }^{5,6}$ BScN, RN, MBE; J Shayo, ${ }^{5}$ BSC, MA, MBE; K Williams, ${ }^{7}$ MSN, CPNP-PC; J F Merz, ${ }^{8}$ MBA, JD, PhD; C M Ulrich, ${ }^{9}$ PhD, RN \\ 'Department of Surgery, School of Medicine, Muhimbili University of Health and Allied Sciences (MUHAS), Dar es Salaam, Tanzania \\ 2 PhD Student, Department of Haematology and Blood Transfusion, School of Medicine, Muhimbili University of Health and Allied Sciences (MUHAS), \\ Dar es Salaam, Tanzania \\ ${ }^{3}$ Geisel School of Medicine, Dartmouth College, New Hampshire, United States \\ ${ }^{4}$ Emeritus Professor, Harvard School of Medicine, Boston, United States \\ ${ }^{5}$ Department of Bioethics and Health Professionalism, Muhimbili University of Health and Allied Sciences (MUHAS), Dar es Salaam, Tanzania \\ ${ }^{6}$ Nursing Department of Management, Muhimbili University of Health and Allied Sciences, Dar es Salaam, Tanzania \\ 7 University of Pennsylvania School of Nursing, Philadelphia, United States \\ ${ }^{8}$ Department of Medical Ethics and Health Policy, Perelman School of Medicine, Philadelphia, United States \\ ${ }^{9}$ Lillian S Brunner Chair, University of Pennsylvania School of Nursing, Philadelphia, United States
}

Corresponding author: C M Ulrich (culrich@nursing.upenn.edu)

\begin{abstract}
Ethical issues are common in the global community. The shortage of human and medical resources when working with vulnerable populations requires institutional support to address the challenges that often arise in the patient-provider relationship. The 2014 Dartmouth/Penn Research Ethics Training and Program Development for Tanzania (DPRET) workshop centred on discussions about research and clinical ethics issues unique to Tanzanian healthcare providers. This article discusses some of the ethical challenges that workshop participants reported in their day-to-day work life with patients and families, such as truth-telling, disagreements over treatment plans and patient distrust of local physicians and hospital staff, among others. The Tanzanian participants recognised the need for supportive mechanisms within their local hospital environments. Further dialogue and research on the development of institutional ethics committees within hospital systems is critically needed so that healthcare providers can meet their ethical and professional obligations to patients and families and address ethical conflicts that arise in a timely and productive fashion.
\end{abstract}

S Afr J Bioethics Law 2018;11(2):75-79. DOI:10.7196/SAJBL.2018.v11i2.639

Like researchers, clinicians (i.e. nurses, physicians and other healthcare providers) face complex ethical issues in the delivery of patient care in Tanzania. However, there are few resources to aid those faced with making difficult ethical decisions in clinical practice. Healthcare practitioners must individually handle clinical ethics problems that arise within their respective clinics, hospitals or other settings, making decisions based on their own beliefs about what is morally right or wrong, when no ethics committee or ethics support system exists. This can lead to frustration, dissatisfaction and high turnover of the clinicians who are critical to the country's infrastructure and overall quality of healthcare.

Healthcare ethics committees (HECS) are common within US hospital-based institutions, and assist clinicians, patients, families and others with difficult ethical problems that arise in patient care delivery. An analysis of data from 300 HEC chairpersons in the USA, for example, showed that the most successful functions reported by HEC members were patient, family, community and provider education and conflict mediation. ${ }^{[1]}$ Additionally, the most frequent clinical issue that HEC chairpersons reported, and also the one best handled, was that of making end-of-life decisions (including 'do not resuscitate', advance directives and withdrawing and withholding treatment), which remains a ubiquitous issue in many healthcare institutions. In their survey of 600 US general hospitals, Fox et al. ${ }^{[2]}$ also identified reported goals of ethics consultations as intervening to protect patients' rights, addressing conflicts, discussing ethical issues with staff and providing moral support as needed, among other issues. Moreover, in a randomised trial of 551 patient cases in the USA in 2001, the majority (87\%) of healthcare professionals, patients and families claimed that ethics consultations in the intensive care unit (ICU) were beneficial in alleviating conflict, and perceived as facilitative. ${ }^{[3]}$ The majority of patients and surrogates also indicated that they would seek an ethics consultation again, and recommend it to others. This same study investigated the effects of ethics committees on duration of stay in the ICU, and on prolonged non- 
beneficial life-sustaining treatments. Ethics consultations reduced hospital and ICU stays, as well as aggressive treatments in patients who were not likely to survive to discharge.

With the healthcare-worker-to-population ratio in Tanzania far below the World Health Organization (WHO) guidelines, ${ }^{[4]}$ and the Ministry of Health and Social Welfare ${ }^{[5]}$ reporting a workforce crisis in Tanzania in $2008,{ }^{[6]}$ having limited or no processes or supportive mechanisms to help assist Tanzanian clinicians address ethical problems and concerns, impacts the sustainability of the workforce, as well as satisfaction with care delivery, as evidenced from research on the value of HECs' and ethics consultations.

Sirili et al. ${ }^{[7]}$ reported a significant human-resource health shortage (e.g. of nurses, physicians, dentists, pharmacists and support staff) in both private and public Tanzanian hospitals (87.5\% and $67 \%$, respectively). Furthermore, a large discrepancy exists in healthcare worker distribution, as slightly more than half of physicians (52\%) work in the urban Dar es Salaam area, although the majority of the Tanzanian population resides in rural communities. ${ }^{[8]}$ Moreover, 'expanding the number of doctors from the most recent estimate of 0.31 doctors per 10000 population to meet the demands of the population' is critical. ${ }^{[8]}$ The strained and unbalanced healthcare worker situation demands that greater attention be given to addressing the ethical issues that affect clinical dissatisfaction and retention, and investing in resources that support the daily life of clinicians, which impacts the patient-provider relationship.

Sabin ${ }^{[9]}$ recently commented that 'the ethical quality of medical care depends as much on the ethics of organisations as the ethics of individuals. For better and worse, the culture and policies of hospitals, group practices, insurers and other health system organisations shape individual clinician-patient relationships.' HECs provide an opportunity for healthcare clinicians to consult with an objective and impartial body on ethical issues of concern, and to help them determine allocation priorities within their work environment. A committee can also help to develop guidelines and set institutional priorities, and support clinicians in day-to-day work-related decisions.

'The overall goal of the Dartmouth/Penn Research Ethics Training and Program Development for Tanzania (DPRET) is to establish new, and strengthen existing, expertise in research ethics among research scientists, faculty, healthcare providers and other professionals at the Muhimbili University of Health and Allied Sciences (MUHAS), and throughout Tanzania.!'10] However, the DPRET programme leaders also recognise that there is a unique interplay between clinical care and research, and so in March 2014, the DPRET conducted a 2-day workshop with nurses, physicians and administrators from research and healthcare institutions in Dar es Salaam, to discuss ethics in research and clinical practice. This article focuses on the clinical concerns of the participants, and the discussions surrounding ethical issues and stressors faced by healthcare clinicians in Tanzanian hospitals and other healthcare settings and the resources needed to mitigate these concerns.

\section{Participants and workshop methods}

We invited a diverse group of healthcare practitioners and administrative personnel to participate in a 2-day workshop in Dar es Salaam Tanzania in March 2014. Representatives from several Tanzanian research and clinical care institutions attended, including the Ocean Road Cancer Institute, Muhimbili National Hospital, MUHAS, International Medical and Technology University, Amana Regional Hospital, Temeke Regional Hospital, Mwananyamala, Muhimbili Orthopaedic Institute and Aga Khan Hospital. The workshop consisted of lecture sessions intermixed with small workgroup activities. Topics addressed over the 2 days included both clinical and research-related topics, such as: international guidance for clinical research practices, challenges to informed consent in clinical research, role and function of healthcare ethics committees, end-of-life issues, critical ethical decision-making for physicians and nurses in clinical practice, ethical dilemmas in patient care and advocacy, the role and function of institutional review boards (IRB), and liaison between the HEC and the IRB/ research ethics board.

There were a total of 45 participants in the workshop (including core faculty of the DPRET and guest lecturers). Of the 45 participants, 27 $(60 \%)$ were primarily trained in medicine, $12(26.6 \%)$ in nursing, $2(4.4 \%)$ in law, $1(2.2 \%)$ in public health and IRB administration and $1(2.2 \%)$ in health sciences and epidemiology; 2 registrants' discipline(s) were not known. Two of the registrants also held administrative positions (dean, executive director of hospital). Members of the DPRET faculty were also trained in bioethics, either holding a Masters of Bioethics degree, a medical degree or a research doctorate. We conducted group discussions with 4 groups of 10 participants each, to gather information on forms of clinical ethics oversight in their respective institutions, using pre-prepared guidelines (several faculty members were also included in these discussions). The workshop participants were given 2 hours to respond to the guideline questions and discuss the questions in their respective groups. Workshop faculty members facilitated the work groups, and at the end of the session, each group compiled the information generated, and presented their thoughts to all participants. Table 1 outlines the clinical ethics-related questions that each group discussed. MUHAS IRB approval was secured for the retrospective analysis of workshop notes and participant evaluations, as well as for a prospective survey aiming to assess clinical ethics oversight in Tanzania.

\section{Results \\ Ethical issues and conflicts experienced by Tanzanian healthcare practitioners}

Participants identified different types of ethical issues that affected their ability to provide quality patient care within their respective institutions (Table 2). These included issues related to truth-telling, cultural views of women in society, allocation of finite resources, end-of-life concerns, patient distrust of local physicians and the use of traditional healers, and the ethics of caring for vulnerable populations. Participants also identified a pattern of ethical conflicts that included delays in treating patients, refusal of patients to be treated, patients not being able to afford treatment and conflicts between families and providers concerning patients' best interests. The most prevalent theme was conflict related to healthcare resource scarcity. This occurred for two reasons - firstly, the inability of patients to pay for treatments rendered, and secondly, healthcare providers' perception that they lacked appropriate resources to treat patients. Other issues noted were conflicting judgments either between patients and clinicians or clinicians and hospital administrators. 


\section{Table 1. Example of group discussion clinical ethics questions}

What ethical issues and ethical conflicts have you encountered in your professional practice as a healthcare provider?

What kind of ethical consultation would be most relevant in handling ethical conflicts in the Tanzanian setting?

Would you combine the functions and roles of an HEC and an IRB under one committee? If not, what would be a practical and convenient mechanism in the Tanzanian context?

Are we even there yet to start thinking of investing in having clinical ethics committees in our settings, considering all the bottlenecks that these committees will be facing? In other words, do Tanzanian hospitals need HECs?

$\mathrm{HEC}=$ healthcare ethics committee; IRB = institutional review board.

\section{Table 2. Common ethical issues experienced by participants}

\section{Ethical issue \\ Patient distrust of local physicians and hospital staff}

Delay in treatment due to family disagreements on patient care needs

Resource allocation

Truth-telling among family members (particularly related to HIV)

End-of-life issues

Moral distress

Role of women in society

Decision-making in clinical care

\section{Case examples}

A 47-year-old with newly diagnosed diabetes mellitus needs to start oral

hypoglycaemic medication, but refuses the medication and decides to go to a local healer. She comes back after 3 months with a gangrenous left leg, and is referred to the local hospital for amputation, but she refuses the amputation. She demands a referral to India for treatment that is deemed irrelevant; she goes back home and dies after a few weeks. The reliance on traditional healers over medical staff can lead to poor patient outcomes when there is distrust of local physicians, and misunderstandings arise.

A 16-year-old presents with osteosarcoma of the knee; the patient and mother agree on an amputation, but the father disagrees. The boy returns to the hospital about 3 months later with the mother, and is in worse condition. They finally agree to surgery, and he is then referred to cancer care.

Hospital medical supplies are limited, and only a single unit of blood is available, but many patients are in need of a blood transfusion. In addition, a single oxygen concentrator is available, but there are 30 patient admissions who need oxygen, with no guide on how to prioritise the oxygen (who to give it to, and why).

A husband is found to be HIV-positive and refuses to tell his wife. The wife then tests positive, and wants to tell the husband, but he indicates that he is giving her antiretrovirals without her knowing that she is infected.

A practitioner is unable to remove a ventilator from a patient who is terminally ill once started, owing to lack of policies/guidelines at end of life.

A woman is bitten by her husband and was taken to the police; the police brought her to the hospital. The husband was aware that the wife had gone to the police. She could not be admitted to the hospital because she did not qualify for admission. The husband was furious and was waiting outside the hospital gate, and soon after the wife and the woman police officer who escorted her were outside the gate, the husband beat the wife, leading to her death. The healthcare team felt as though they did not meet their ethical requirements in protecting the woman from harm, based on the hospital admission guidelines.

A woman with breast cancer has undergone chemotherapy and now needs a mastectomy, but her husband refuses. The woman does not receive the surgery. Therefore, potential harm can come to women based on cultural beliefs about the role of women within a family.

Families are focused on a communal ethic rather than the individual autonomy of a patient, so if family members have different views on medical treatment, it creates distress for healthcare providers. An example of this is that many patients in Tanzania come with their family members to a hospital or other type of health setting, and, for any type of treatment (e.g. family planning, treatment of breast cancer or other types of cancer and many other chronic illnesses), patients will consult with their families and generally will not go against their relatives' wishes in the decisions that are made.

\section{Proposed forms of consultation}

A priority need identified by all participants was the establishment of clinical ethics committees with clinical ethics consultation services. However, they also acknowledged that there was a lack of expertise conversant with clinical ethics. Most participants thought that having a research ethics committee (REC) with combined tasks (being both a REC and an HEC) would not meet their immediate clinical needs. Participants also acknowledged that the decision of whether to establish a clinical ethics committee or REC should be grounded on the primary functions of the institutions. For those Tanzanian 
teaching hospitals whose primary function is to provide patient care, establishing clinical ethics committees and a research ethics committee as a subset of the HEC committee seemed plausible. Participants also made it clear that they had few or no resources to help them resolve ethical problems when they arise. Many of the represented institutions had IRBs and research ethics boards, but not HECs that could specifically address clinical ethics concerns. Participants also recognised the need for a diverse group of members on the ethics committee (e.g. medicine, nursing, pastoral, social work and patient representatives) that could help to shed light on relevant ethical issues that might arise in the day-to-day clinical environment.

\section{Discussion}

This paper highlights the need for further discussions and research on the development of clinical ethics committees in Africa, based on what would fit the Tanzanian context in terms of resources and infrastructure, as well as the policies and regulations available. HECs are institutional resources widely adopted in many parts of the world, where they have proven to be of great value. These committees were created to help resolve clinical ethical dilemmas, and have become instrumental in ongoing clinical decision-making, as well as the development of institutional clinical policies, particularly in the USA. ${ }^{[1]}$ They often provide consultation services and support for clinicians in the day-to-day delivery of patient care, and help to mediate conflicts that arise among clinicians, patients, families and other members of the healthcare team. ${ }^{[12]}$ Indeed, HECs may help to allocate care and resources more effectively, and could be a significant asset to various stakeholders, especially in countries with scarce resources. While there is limited research on the outcomes of HECs, the existing evidence suggests that they can improve the delivery of care and resources, while reducing conflict among providers, patients and families.

Ethical issues were a common theme among our discussion participants. Some of these issues reflect similar concerns to those of healthcare providers from other countries, including end-of-life care, autonomy and protecting patients' rights. ${ }^{[13]}$ Research is needed to identify the frequency and intensity of the ethical issues that arise, and how they affect the general wellbeing of Tanzanian healthcare providers, and whether and how experience with these issues varies by profession, or by sociodemographic or other variables. Research would also be helpful in identifying the mitigating effects of ethics education and other strategies to support healthcare providers.

Moral apathy and distress were expressed by many clinical representatives. This is also consistent with findings from Harrowing and Mill, ${ }^{[14]}$ who examined moral distress among Ugandan nurses providing HIV care. Similarly to our discussants, the nurses in this particular study were distressed by the suffering of their patients, as well as their own emotional angst. This was mostly a result of the lack of resources to provide quality care. However, even amid such distress, these nurses continued to work and to do what they could for patients and families with what was available to them. Consistent exposure to ethical issues without recourse, however, can lead to ethical adaptability in situations where there is a perceived necessity for the act under ethical question. ${ }^{[15]}$ Another possibility is that one simply stops recognising the ethical components of a particular situation, and it fades from our ethical consciousness. ${ }^{[16]}$
Tanzanian physicians also suffer when resources are limited, or when conflicts arise with patients and families. Harris et al. ${ }^{[17]}$ reported on the ethical tensions of physicians in Northern Tanzania, particularly during discussions surrounding the diagnosis and prognosis of cancer with their patients. As Ulrich and Grady ${ }^{[18]}$ so aptly state, 'even when the provider does the best he or she can, it may not feel good enough.' Although many ethical issues remain unresolved, there was an overwhelming sense that more could be done to improve the overall care and wellbeing of not only patients within Tanzania, but also those who provide that care.

Many of the discussants in our workshop deliberated on whether they were ready to establish clinical ethics committees, how they were going to structure them, and what the implications of having these committees within their hospital institutions would mean for all relevant stakeholders. Further research is needed to better understand these issues from the perspective of Tanzanian healthcare clinicians, patients, families and others involved in the delivery of patient care, and how HECs can be instituted within underdeveloped countries such as Tanzania. The DPRET workshop afforded an opportunity to openly bring to the fore sensitive and controversial ethical concerns and issues that arise in everyday clinical practice with our Tanzanian colleagues, and a space for shared discussion. In some ways, it has heightened the need for future conferences in Africa to better understand how traditional African medicine interfaces with Western bioethics and bioethical concepts in caring for patients and families, particularly when responding to certain diseases, such as HIV/AIDS and Ebola. Perhaps there are uniquely African issues for bioethics that require more in-depth dialogue, research and evaluation. Western culture is often focused on the notion of individual autonomy, property and rights, but these values could be more appropriately modified for a more communitarian African culture.

\section{Conclusion}

Research ethics is the most developed aspect of bioethics in Africa. In Tanzania, many health research institutions and even medical institutions have well-established RECs or IRBs whose primary role is to protect human subjects who are participating in research. Institutions such as the National Institute for Medical Research (NIMR), Ifakara Health Institute and Kilimanjaro Christian Medical College have RECs. There are also guidelines developed locally by the Tanzania National Health Research Forum, and by NIMR, to guide researchers as to how to conduct ethical research. However, a new direction is now needed; there is a pressing need for clinical ethics education, and committees that support clinicians in the ethical issues that arise in their day-to-day practice. We acknowledge that the initial formation of Tanzanian ethics committees needs to be locally grounded, through understanding both the local healthcare system and the resources available. However, as in many parts of Africa south of the Sahara, the greatest challenge in achieving this goal is the limited resources available. Ethics committees will require members who have some clinical ethics education, which will require additional funds for training.

The healthcare system is currently burdened with a number of issues, including fewer healthcare professionals than required, and so convincing hospital administrators of this need will be a challenge. In the long term, however, this might serve as a positive step to improve 
the satisfaction and retention of the Tanzanian healthcare workforce. Bioethics itself is also a relatively new concept in sub-Saharan Africa, and therefore introducing clinical ethics consultations will take time and effort on the part of those newly trained in bioethics within the country (2 nurses, 1 physician, 1 IRB administrator and 1 lawyer) through the DPRET. There is an immediate need to support healthcare providers in Tanzania, as they continue to face ethical challenges as part of their daily care for the citizens within their country.

Acknowledgements. We acknowledge Drs Christine Grady and Jeremy Sugarman for their thoughtful comments on this work, as well as all participants who were part of the seminar and provided thoughtfu dialogue on the ethical challenges of providing clinical care.

Author contributions. All authors contributed to the substance of the manuscript.

Funding. We acknowledge funding from the National Institutes of Health, Fogarty Grant no. 1R25TW007693.

Conflicts of interest. None.

1. McGee G, Spanogle JP, Caplan AL, Penny D, Asch DA. Successes and failures of hospital ethics committees: A national survey of ethics committee chairs. Camb Q Healthc Ethic 2002;11(1):87-93. https://www.cambridge.org/core/journals/ cambridge-quarterly-of-healthcare-ethics/article/successes-and-failures-of hospital-ethics-committees-a-national-survey-of-ethics-committee-chairs/77D7E E4DEF73A39BD8141B4EE9A7EB8A (accessed 14 March 2016).

2. Fox $E_{1}$ Myers $S$, Pearlman RA. Ethics consultation in United States hospitals: A national survey. Am J Bioethics 2007;7(2):13-25. https://doi. org/10.1080/15265160601109085

3. Schneiderman LJ, Gilmer T, Teetzel HD, et al. Effect of ethics consultations on nonbeneficial life-sustaining treatments in the intensive care setting - a randomised controlled trial. JAMA 2003;290(9):1166-1172. https://doi.org/10.1001/ jama.290.9.1166

4. World Health Organization. Global Health Observatory. Geneva: WHO, 2012 http:// www.who.int/gho/health_workforce/physicians density/en/ (accessed 9 May 2016).

5. United Republic of Tanzania Ministry of Health and Social Welfare. Human Resource for Health Country Profile 2012/2013. Dar es Salaam: Ministry of Health and Social Welfare, 2013. https://www.jica.go.jp/project/tanzania/006/materials/ ku57pq00001x6jyl-att/country_profile_2013.pdf (accessed 18 March 2016).
6. Kwesigabo G, Mwangu MA, Kakoko DC, et al. Tanzania's health system and workforce crisis. J Public Health Pol 2012;33(Suppl 1):S35-S44. https://doi. org/10.1057/jphp.2012.55

7. Sirili N, Kiwara A, Nyongole O, Frumence GSA, Hurtig A. Addressing the human resource for health crisis in Tanzania: The lost in translation syndrome. Tanzan $J$ Health Res 2014;16(2):1-9. http://www.bioline.org.br/pdf?th14013 (accessed 206 Mar 18). https://doi.org/10.4314/thrb.v16i2.6

8. Goodell AJ, Kahn JG, Ndeki SS, Kaale E, Kaaya EE, Macfarlane SB. Modeling solutions to Tanzania's physician workforce challenge. Glob Health Action 2016;9:31597. https://doi.org/10.3402/gha.v9.31597

9. Sabin JE. How can clinical ethics committees take on organisational ethics? Some practical suggestions. J Clin Ethics 2016;27(2):111-116.

10. Waddell R, Aboud M. Dartmouth/MUHAS research ethics training and program development for Tanzania, [R25TW007693]. Tanzania: National Institutes of Health, Fogarty; 2011-2016.

11. McGee G, Caplan AL, Spanogle JP, Asch DA. A national study of ethics committees. Am J Bioeth 2001;1(4):60-64. https://doi.org/10.1162/152651601317139531

12. Geppert CM, Shelton W. Healthcare ethics committees as mediators of social values and the culture of medicine. AMA J Ethics 2016;18(5):534-539. https://doi. org/10.1001/journalofethics.2016.18.5.msoc1-1605

13. Ulrich CM, Taylor C, Soeken K, et al. Everyday ethics: Ethical issues and stress in nursing practice. J Adv Nurs 2010;66(11):2510-2519. https://doi.org/10.1111/ j.1365-2648.2010.05425.x

14. Harrowing JN, Mill J. Moral distress among Ugandan nurses providing HIV care: A critical ethnography. Int J Nurs Stud 2010;47(6):723-731. https://doi. org/10.1016/j.ijnurstu.2009.11.010

15. Wynia MK, Cummins DS, VanGeest JB, Wilson IB. Physician manipulation of reimbursement rules for patients - between a rock and a hard place. JAMA 2000;283(14):1858-1865. https://doi.org/10.1001/jama.283.14.1858

16. Bazerman MH, Tenbrunsel A. Blind Spots: Why We Fail to Do What is Right and What to Do About It. Princeton: Princeton University Press, 2001.

17. Harris JJ, Shao J, Sugarman J. Disclosure of cancer diagnosis and prognosis in Northern Tanzania. Soc Sci Med 2003;56(5):905-913. https://doi.org/10.1016/ s0277-9536(02)00090-4

18. Ulrich CM, Grady C. Doing 'good' with limited resources: Is it good enough in the provision of quality clinical care? Clin Scholars Rev 2009;2(1):5-7. https://doi. org/10.1891/1939-2095.2.1.5

Accepted 9 May 2018 